\title{
Highly absorbed X-ray binaries in the Small Magellanic Cloud ${ }^{\star}$
}

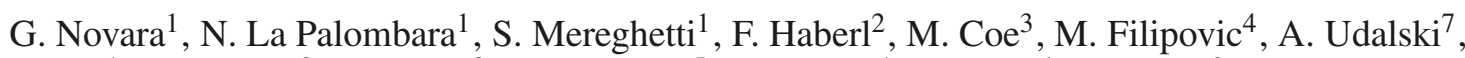

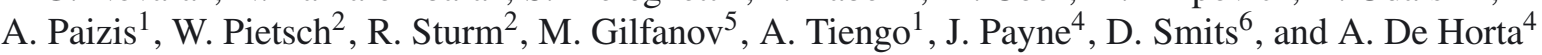 \\ 1 INAF - IASF, Istituto di Astrofisica Spaziale e Fisica Cosmica “G.Occhialini”, via Bassini 15, 20133 Milano, Italy \\ e-mail: novara@iasf-milano.inaf.it \\ 2 Max-Planck-Institut für extraterrestrische Physik, Giessenbachstraße, 85748 Garching, Germany \\ 3 School of Physics and Astronomy, University of Southampton, SO17 1BJ, UK \\ ${ }^{4}$ University of Western Sydney, Locked Bag 1797, Penrith South DC, NSW 1797, Australia \\ 5 Max-Planck-Institut für Astrophysik, Karl-Schwarzschild-Straße 1, 85741 Garching, Germany \\ ${ }^{6}$ Department of Mathematical Sciences, University of South Africa, UNISA, Pretoria 0003, South Africa \\ 7 Warsaw University Observatory, Aleje Ujazdowskie 4, 00-478 Warsaw, Poland
}

Received 31 March 2011 / Accepted 20 June 2011

\begin{abstract}
Many of the high mass X-ray binaries (HMXRBs) discovered in recent years in our Galaxy are characterized by a high absorption, most likely intrinsic to the system, that can impede their detection at the softest X-ray energies. Exploiting the good coverage obtained with sensitive XMM-Newton observations, we have undertaken a search for highly absorbed X-ray sources in the Small Magellanic Cloud (SMC), which is known to contain a large number of HMXRBs. After a systematic analysis of 62 XMM-Newton SMC observations, we obtained a sample of 30 sources with evidence of an equivalent hydrogen column density larger than $3 \times 10^{23} \mathrm{~cm}^{-2}$. Five of these sources are clearly identified as HMXRBs, four being previously known (including three X-ray pulsars) and one, XMMU J005605.8-720012, being reported here for the first time. For the latter, we present optical spectroscopy confirming the association with a Be star in the SMC. The other sources in our sample have optical counterparts fainter than magnitude $\sim 16$ in the $V$ band, and many have possible NIR counterparts consistent with highly reddened early-type stars in the SMC. While their number is broadly consistent with the expected population of background highly absorbed active galactic nuclei, a few of them could be HMXRBs in which an early-type companion is severely reddened by local material.
\end{abstract}

Key words. Magellanic Clouds - X-rays: general - X-rays: galaxies - X-rays: binaries

\section{Introduction}

Many observations of the Small Magellanic Cloud (SMC) in the $\mathrm{X}$-ray energy band have led to the discovery of a large number of high mass X-ray binaries (HMXRBs). The number of known HMXRBs in the SMC (about one hundred, Liu et al. 2005 ) is much larger than expected by scaling the number of these sources seen in the Milky Way according to the mass ratio of the two galaxies $\left(M_{\mathrm{MW}} / M_{\mathrm{SMC}} \sim 50\right)$. This has been interpreted as evidence of a recent episode of star formation in the SMC (Majid et al. 2004; Shtykovskiy \& Gilfanov 2007; Antoniou et al. 2010). It is remarkable that the SMC contains only one supergiant system, SMC X-1. All the other HMXRBs in the SMC consist of neutron stars accreting from Be type companions. Be $\mathrm{X}$-ray binaries also constitute the largest class of HMXRBs in our Galaxy, but the relative number of supergiant systems is much higher than in the SMC, since 30 of the 114 Galactic HMXRBs (Liu et al. 2006) are confirmed or suspected supergiant systems.

Many new Galactic HMXRBs have been discovered in the past few years with the INTEGRAL satellite, thanks to high sensitivity in the hard X-ray range, coupled with an extensive monitoring of the Galactic plane. These observations led to the recognition of the new class of supergiant fast X-ray transients (SFXT) and to the discovery of persistent supergiant systems characterized by a high absorption (equivalent column density

* Table 1 is available in electronic form at http://www. aanda.org above a few $10^{23} \mathrm{~cm}^{-2}$ ), which escaped an earlier discovery because the high absorption severely suppresses their flux below $10 \mathrm{keV}$ (see, e.g., Sidoli 2010, for a recent review).

To search for highly absorbed HMXRBs in the SMC, we carried out a dedicated analysis of the XMM-Newton data collected during the large program for the SMC survey performed by Haberl \& Pietsch (2008) and other observations of SMC targets. In Sect. 2, we describe the X-ray observations and data processing and in Sect. 3 we describe the source detection and selection criteria that led us to identify a sample of 30 highly absorbed sources. Five of them are confirmed HMXRBs, including four that were already known as X-ray binaries (Sect. 4). In Sect. 5, we present optical spectroscopy of the new source, demonstrating that it is a Be system in the SMC. We finally discuss our results in Sect. 6 .

\section{X-ray observations and data processing}

We analyzed the $62 X M M$-Newton pointings of the SMC performed from 2001 May 31 to 2010 March 16 listed in Table 1. The three EPIC focal plane cameras (Turner et al. 2001; Strüder et al. 2001) were operated in standard full frame mode.

The data of each camera were processed independently using the standard XMM-Newton Science Analysis Software (SAS, v.10.0.0). We first used the tasks emproc and epproc to obtain the MOS and $p n$ event files of each pointing. We then filtered out time intervals affected by high instrumental background induced 


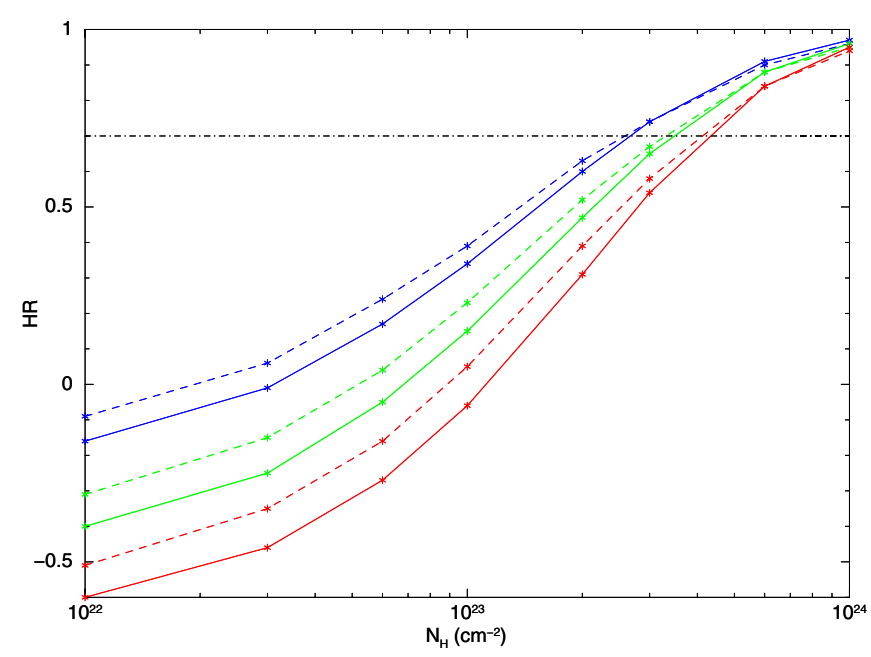

Fig. 1. Expected hardness ratio (of the count rates in the energy ranges $1-3$ to $3-10 \mathrm{keV}$ ) as a function of the column density. Solid and dashed lines refer to the $p n$ and the MOS cameras, respectively. Red, green, and blue lines refer to power-law spectra with photon indices of 2 , 1.5 , and 1 , respectively. The horizontal line at $\mathrm{HR}=0.7$ represents the threshold value used to select highly absorbed sources.

by flares of soft protons (with energies below a few hundred $\mathrm{keV}$ ). To avoid contributions from genuine $\mathrm{X}$-ray source variability, this was done by examining light curves binned at $100 \mathrm{~s}$ for events with energies above $10 \mathrm{keV}$ and PATTERN $\leq 4$ or 0 for, respectively, the MOS peripheral CCDs and the whole $p n$ camera; the good time intervals (GTI) were selected by adopting different count-rate thresholds for the different pointings and instruments. By selecting only events within GTI, we finally obtained three "clean" event lists for each observation (i.e. two for the MOS and one for the $p n$ cameras), whose exposure times are reported in Table 1. The total net exposure times are respectively 1572 and $1598 \mathrm{ks}$ for the MOS cameras, and $1361 \mathrm{ks}$ for the pn one.

\section{Source detection and selection criteria}

We optimized our source detection strategy to select highly absorbed X-ray sources. To this aim, we simulated the expected EPIC count distributions for absorbed power-law spectra, with photon-index $\Gamma$ in the range $1-2$. We used two absorption components: the first one, with column density fixed at $6 \times 10^{20} \mathrm{~cm}^{-2}$ and elemental abundances from Wilms et al. (2000), accounts for the foreground absorption in our Galaxy; for the second one, which accounts for the absorption in the SMC and is intrinsic to the source, we considered $N_{\mathrm{H}}$ values in the range $10^{22}-10^{24} \mathrm{~cm}^{-2}$, and metal abundances of 0.2 , as is typical of the SMC (Russell \& Dopita 1992). We defined the hardness ratio $\mathrm{HR}=(H-S) /(H+S))$, based on the count rates in the soft $(\mathrm{S}$, 1-3 keV) and hard ( $\mathrm{H}, 3-10 \mathrm{keV})$ energy ranges. These simulations showed that, for $\Gamma=2$ and $N_{\mathrm{H}}>3 \times 10^{23} \mathrm{~cm}^{-2}$, the fraction of the detected source counts with energies above $3 \mathrm{keV}$ is at least $70 \%$, and that this fraction is even higher for lower values of $\Gamma$. In Fig. 1, we report the estimated HR values as a function of $N_{\mathrm{H}}$, for both the $p n$ and MOS cameras and different values of $\Gamma$. On the basis of these simulations, we adopted a HR threshold value of 0.7 , in order to select sources with $N_{\mathrm{H}}>3 \times 10^{23} \mathrm{~cm}^{-2}$.

For each observation in Table 1, we used the cleaned event file to produce merged images from the three cameras in the 1-3 and 3-10 keV energy ranges, according to the procedure

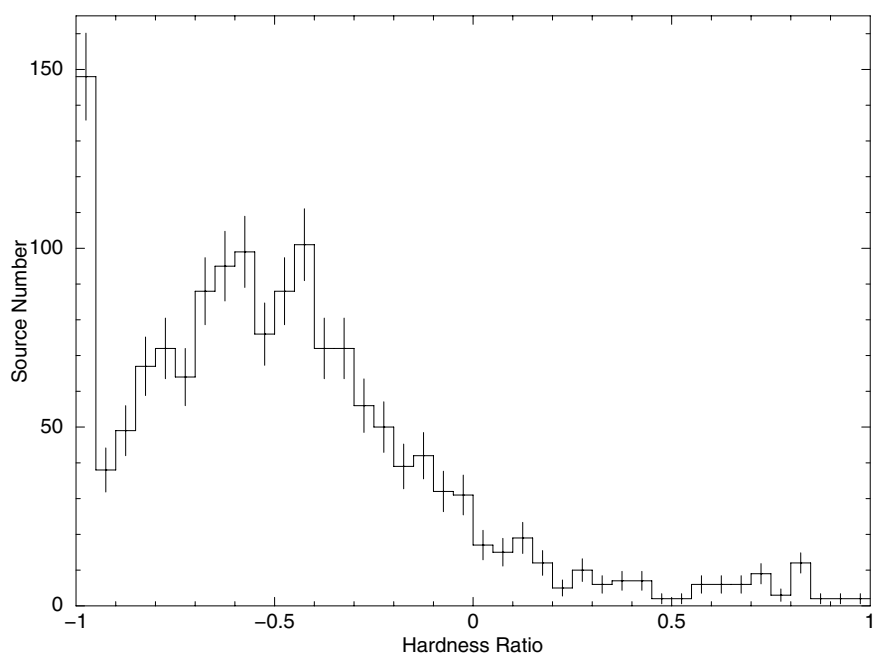

Fig. 2. Hardness-ratio distribution of the detected X-ray sources.

described in detail in Baldi et al. (2002). For the 3-10 keV pn images, we excluded the energy range $7.8-8.2 \mathrm{keV}$, in order to reduce the instrumental background caused by the $\mathrm{Cu}$ line. For each image, we also produced the corresponding merged exposure map, which accounts for mirror vignetting and effective field of view. The total count-rate-to-flux conversion factors were obtained as a mean of the MOS1/MOS2/pn factors, weighted on the individual exposures of the three cameras. The source detection was based on a maximum likelihood technique, as described in detail in Novara et al. (2006), and used background maps produced with the correction algorithm described in Baldi et al. (2002). For each energy band, we only selected sources with a final detection likelihood $-\ln P>8.5$ (where $P$ is the probability of a false detection due to a Poissonian random fluctuation of the background). This likelihood threshold corresponds to a $\sim 3 \sigma$ detection. After manually removing a few spurious detections caused, for instance, by events falling close to the CCD edges, we obtained a final master list containing $\simeq 1500$ sources. For each source, the master list provides various parameters including the detector and sky coordinates, the effective exposure time, the total counts, count-rate and errors in the different energy ranges, and the detection likelihood.

\section{Results}

In Fig. 2, we report the HR distribution of all the detected sources. This figure illustrates that most sources have $\mathrm{HR}<0$, with a peak around -0.5 , hence they are characterized by nonabsorbed soft spectra. On the other hand, only very few sources have high HR values. In our final source list, there are 30 objects with $\mathrm{HR}>0.7$, which we consider as highly absorbed sources with $N_{\mathrm{H}}>3 \times 10^{23} \mathrm{~cm}^{-2}$; given the HR distribution, we expect that, even if we take into account the HR errors, only a very few additional sources would have HR $>0.7$. The sky coordinates of these 30 objects, corrected for the satellite pointing uncertainty, are listed in Table 2 . The astrometric correction of the $\mathrm{X}$-ray images was done by cross-matching the brightest sources of each field with the Magellanic Clouds Photometric Survey optical catalogue (MCPS, Zaritsky et al. 2002), and selecting the $\mathrm{X}$-ray sources with a single, clearly evident optical counterpart within a $3^{\prime \prime}$ error radius. We then used the IRAF task geomap to compute the linear transformation between the X-ray and optical coordinates of these reference sources. 
G. Novara et al.: Highly-absorbed X-ray binaries in the SMC

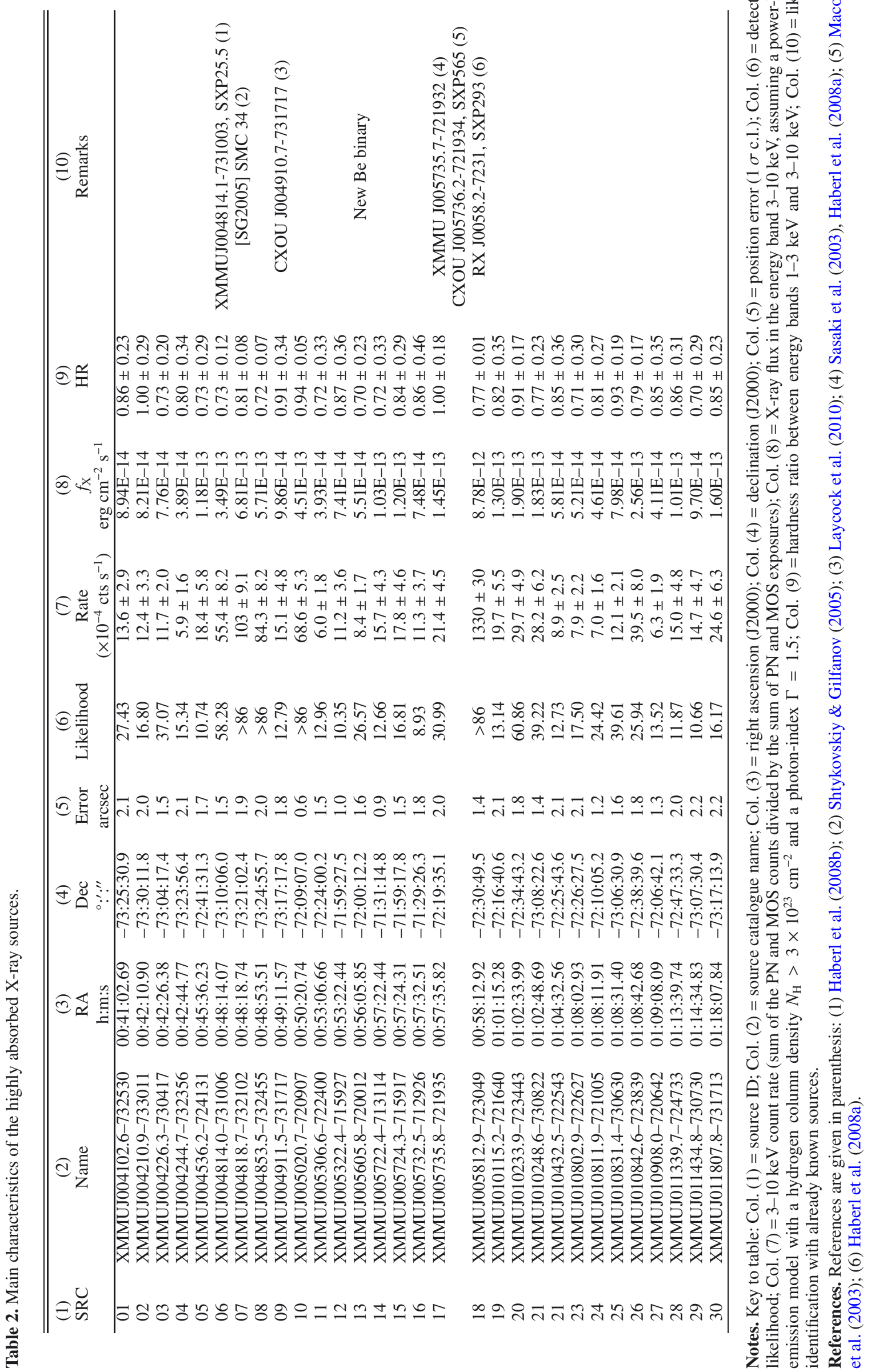


The calculated X-ray-to-optical coordinate transformations have rms fit residuals between 0.43 and $1 . .85$ at $1 \sigma$ c.1., with an average value of 1 .' 1 ; they correspond to the (radial) systematic astrometric errors in the corrected observations. These transformations were then used by the IRAF task geoxytran to obtain the corrected X-ray coordinates given in Table 2. The total uncertainty on the X-ray position of each source is given by the sum in quadrature of the calculated systematic astrometric error in the observation and the measured statistical error of the source itself.

We also list in Table 2 the hardness ratios, count rates, and fluxes of the sources. The fluxes refer to the 3-10 keV energy range, and have been computed assuming a power-law with photon index $\Gamma=1.5$ and $N_{\mathrm{H}}=3 \times 10^{23} \mathrm{~cm}^{-2}$. The brightest source has a absorbed flux of $9 \times 10^{-12} \mathrm{erg} \mathrm{cm}^{-2} \mathrm{~s}^{-1}$, while the faintest sources in our sample have fluxes of $\sim 4 \times 10^{-14} \mathrm{erg} \mathrm{cm}^{-2} \mathrm{~s}^{-1}$ corresponding to $\sim 2 \times 10^{34} \mathrm{erg} \mathrm{s}^{-1}$, assuming a SMC distance of $60 \mathrm{kpc}$ (Hilditch et al. 2005).

Some of our selected sources were already reported in other studies of the SMC, in these cases we give in the table their original name and the relevant references. Four sources, including three pulsars, were already known as HMXRBs: SXP25.5, SMC34, SXP565, and SXP293. We searched for possible counterparts of our highly absorbed sources using the MCPS catalogue, which is based on observations performed in the $U, B$, $V$, and $I$ filters between November 1996 and December 1999 (Zaritsky et al. 2002), and the InfraRed Survey Facility (IRSF) Magellanic Cloud catalogue (Kato et al. 2007) for the NIR (J, H, and $K$ filters).

For each source, we report in Table 3 the optical and NIR properties of the brightest object in the $K$ band present within a distance equal to two times the $1 \sigma$ position error (with only five exceptions, marked in the table, the brightest object in $K$ is also the brightest in $V$ ). For five sources, no catalogued optical or NIR counterparts were found to limits as faint as of $V \sim 23 \mathrm{mag}$ and $K \sim 17$ mag.

A plot of the NIR versus optical colors shows that the four already known HMXRBs of our sample are located in the region corresponding to $J-K<0.5 \mathrm{mag}$ and $B-V<0.5 \mathrm{mag}$ (see Fig. 3). As discussed in Sect. 5, the other source falling in this region (source \#13) is a new Be binary system in the SMC. Three of these sources are well above the position of the mainsequence stars (whose colors are taken from Johnson 1966): if they are affected by an IR excess (which could be due to the decretion disk of the Be star), their optical/NIR colors are consistent with early-type stars with moderate optical reddenings $\left(A_{V} \sim 2-3 \mathrm{mag}\right)$. On the other hand, HMXRBs in which the optical companion is also affected by a high local absorption would show much redder colors, as seen in several of the new HMXRBs discovered by INTEGRAL in our Galaxy. Therefore, we cannot exclude that some of the other sources plotted in Fig. 3, or sources for which no optical and NIR counterparts have been detected, are also highly absorbed HMXRBs. The five sources at the top of the plot are far from the main-sequence stars, therefore it is difficult to suggest a reliable classification of them; this is particularly true for the three sources shown as open circles, for which a different counterpart could also be suggested. These five sources could be early-type stars only if they are characterized by both a large IR excess and a high reddening. On the other hand, since for AGNs $J-K>0.5$ (Kouzuma \& Yamaoka 2010) and $-0.4<B-V<0.7$ (Hatziminaoglou et al. 2002), these sources could be more probably classified as reddened AGNs.

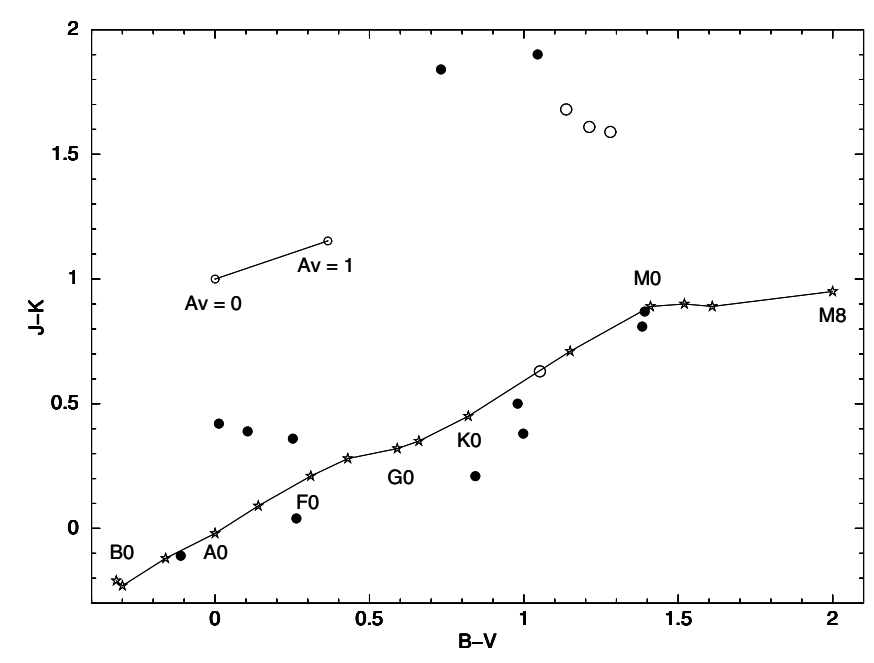

Fig. 3. Color indices of the candidate counterparts of the highly absorbed X-ray sources of our sample. For the sources reported with a filled circle, the brightest optical and infrared candidate counterparts are coincident, while the sources reported as an open circle correspond to those with the "a" flag in Table 3: in this case, there is also a possible alternative counterpart, with a slightly brighter $V$ magnitude, to the selected infrared counterpart. We also indicate the main-sequence stars and a bar which shows the reddening corresponding to $A_{V}=1 \mathrm{mag}$.

To search for possible periodicities in any optical counterparts close to the XMM-Newton sources, data from OGLE III were acquired for all objects within $4^{\prime \prime}$ of the 30 XMM-Newton position. We applied a Lomb-Scargle analysis to a total of 129 lightcurves, searching for periods in the range 2-200 days. Periods shorter than 2 days were avoided because they are getting close to the average daily sampling of the OGLE data, and periods longer than 200 days approach the annual sampling patterns. In each case, the data were first de-trended using a polynomial of order three before being searched. In some cases, there were many optical counterparts; conversely, other sources had no optical objects within the search zone.

Significant periodicities were found for four sources (three of which were already known) and tentative periods for an additional three objects (see Table 4). That three previously known periods were "re-discovered" in this search reassures us that the techniques used are effective in identifying possible periods. The one new significant detection (source number 19) shows a modulation that is extremely sinusoidal in nature when the data are folded at the period of 199.6 days. This modulation shape is not normal for a long-period binary modulation, which tends to reveal short outbursts coincident with the periastron passage of the neutron star (see, for example, Coe \& Edge 2004). Such smooth sinusoidal modulations tend to be associated with pulsations in stars. A likely possibility is that it could be an Non-Radial Pulsations (NRP) in a B-type star with a period very close to the one day sampling, with the result that the signal at 199.6 days is the beat frequency of the true pulse period and the sampling period.

\section{XMMU J005605.8-720012: a new Be HMXRB in the SMC}

Source \#13 was detected with a total of 68 counts in the hard energy range 3-10 keV and a HR value of 0.71 , just above our threshold. It has a relatively bright $(V=16.71 \mathrm{mag})$ optical counterpart, with color $B-V=-0.12 \mathrm{mag}$, and with a quality 
G. Novara et al.: Highly-absorbed X-ray binaries in the SMC

Table 3. Candidate optical-IR counterparts of the highly absorbed X-ray sources.

\begin{tabular}{|c|c|c|c|c|c|c|}
\hline (1) & (2) & (3) & (4) & (5) & (6) & (7) \\
\hline ID & NAME & $\begin{array}{c}V \\
\text { mag }\end{array}$ & $\begin{array}{l}f_{\mathrm{X}} / f_{V} \\
\log _{10}\end{array}$ & $\begin{array}{c}K \\
\mathrm{mag}\end{array}$ & $\begin{array}{c}B-V \\
\mathrm{mag}\end{array}$ & $\begin{array}{c}J-K \\
\mathrm{mag}\end{array}$ \\
\hline 01 & IRSF 00410291-7325332 & $20.79^{a}$ & 0.76 & 16.68 & 1.21 & 1.61 \\
\hline 02 & IRSF $00421082-7330127$ & $19.31^{a}$ & 0.13 & 17.00 & 1.05 & 0.63 \\
\hline 03 & IRSF 00422638-7304184 & $>23$ & $>1.58$ & 15.65 & - & 2.02 \\
\hline 04 & IRSF 00424550-7323553 & 17.05 & -1.09 & 13.90 & 1.39 & 0.87 \\
\hline 05 & IRSF 00453646-7241343 & 19.43 & 0.34 & 15.72 & 0.73 & 1.84 \\
\hline 06 & IRSF 00481410-7310040 & 15.30 & -0.85 & 15.64 & 0.26 & 0.04 \\
\hline 07 & IRSF 00481871-7321000 & 16.18 & -0.21 & 15.29 & 0.25 & 0.36 \\
\hline 08 & IRSF 00485330-7324574 & $>23^{a}$ & $>2.44$ & 15.55 & - & 1.30 \\
\hline 09 & IRSF 00491089-7317172 & 18.80 & 0.01 & 15.95 & 1.00 & 0.38 \\
\hline 10 & IRSF 00502062-7209073 & $>23$ & 2.34 & 16.86 & - & 1.65 \\
\hline 11 & $?$ & $>23$ & $>1.28$ & $>17$ & - & - \\
\hline 12 & MCPS 2493874 & 20.74 & 0.65 & $>17$ & 0.47 & - \\
\hline 13 & IRSF 00560575-7200118 & 16.71 & -1.08 & 17.18 & -0.11 & -0.11 \\
\hline 14 & ? & $>23$ & $>1.70$ & $>17$ & - & - \\
\hline 15 & MCPS 3076421 & 21.42 & 1.14 & $>17$ & 0.35 & - \\
\hline 16 & ? & $>23$ & $>1.56$ & $>17$ & - & - \\
\hline 17 & IRSF 00573600-7219342 & 15.99 & -0.95 & 15.35 & 0.01 & 0.42 \\
\hline 18 & IRSF 00581258-7230488 & 14.87 & 0.38 & 14.14 & 0.10 & 0.39 \\
\hline 19 & IRSF 01011529-7216374 & $19.23^{a}$ & 0.30 & 14.69 & 1.28 & 1.59 \\
\hline 20 & IRSF $01023342-7234421$ & $19.64^{a}$ & 0.62 & 16.09 & 1.14 & 1.68 \\
\hline 21 & IRSF 01024839-7308213 & 20.99 & 1.15 & 17.08 & 1.04 & 1.90 \\
\hline 22 & IRSF 01043305-7225407 & 16.23 & -1.25 & 13.12 & 1.38 & 0.81 \\
\hline 23 & MCPS 4411974 & 20.01 & 0.21 & $>17$ & 0.04 & - \\
\hline 24 & IRSF 01081153-7210048 & $>23$ & $>1.35$ & 17.26 & - & 1.61 \\
\hline 25 & IRSF $01083147-7306325$ & 18.00 & -0.41 & 15.84 & 0.98 & 0.50 \\
\hline 26 & IRSF 01084310-7238375 & 19.53 & 0.71 & 17.84 & 0.84 & 0.21 \\
\hline 27 & IRSF $01090778-7206438$ & 21.13 & 0.55 & $>17$ & 0.57 & - \\
\hline 28 & MCPS 4879163 & 22.36 & 1.44 & $>17$ & 0.56 & - \\
\hline 29 & ? & $>23$ & $>1.67$ & $>17$ & - & - \\
\hline 30 & ? & $>23$ & $>1.89$ & $>17$ & - & - \\
\hline
\end{tabular}

Notes. ${ }^{(a)}$ For this object there is a possible counterpart with a slightly brighter $V$ magnitude.

Key to table: Col. (1) = source ID; Col. (2) = name of the brightest infrared (IRFS sources, Kato et al. 2007) or optical (MCPS sources, Zaritsky et al. 2002) candidate counterpart within the X-ray error circle (a "?" is reported when no counterpart is found); Col. (3)=V magnitude; Col. (4) = X-ray-to-optical flux ratio (in $\log _{10}$ units); Col. (5) $=K$ magnitude; Col. (6) $=B-V$ color index; Col. (7) $=J-K$ color index.

Table 4. List of OGLE III objects within 4" of an XMM-Newton position that show evidence of a periodic signal.

\begin{tabular}{lcccccc}
\hline \hline XMM ID & OGLE III ID & $V$ & $I$ & LS power & Period & Comments \\
\hline 2 & 22605 & 21.3 & 20.3 & 12 & 2.16 days & Tentative \\
6 & 50768 & 15.8 & 15.7 & 14 & 22.5 days & SXP25.5 (Rajoelimanana et al. 2011) \\
8 & 20669 & $?$ & 21.0 & 13 & 4.08 days & Tentative \\
17 & 27992 & 16.1 & 15.8 & 26 & 157.2 days & SXP565 (Bird et al. 2011) \\
18 & 19191 & 14.9 & 14.6 & 200 & 59.7 days & SXP293 (Schmidtke et al. 2004) \\
20 & 43690 & 20.8 & 21.0 & 15 & 5.96 days & Tentative \\
26 & 22676 & 19.6 & 18.6 & 23 & 199.6 days & Possible new period \\
\hline
\end{tabular}

flag in the MCPS indicating a successful fit with a stellar atmosphere model. It has an unabsorbed luminosity, in the energy range $3-10 \mathrm{keV}$, of $4.4 \times 10^{34} \mathrm{erg} \mathrm{s}^{-1}$.

Optical spectral observations of source \#13 were obtained in September 2010, using the 1.9-m telescope and the Cassegrain spectrograph at the South African Astronomical Observatory (SAAO) in Sutherland. We used grating number 7 (300 lines per mm) to obtain spectra between $3700 \AA$ and $7700 \AA$ at a resolution of $5 \AA$. For these, the slit size was 1 '. $5 \times 1$ 1.5. Exposure times were limited to $900 \mathrm{~s}$ with a positional accuracy of $<1^{\prime \prime}$. Data reduction included bias subtraction and flat-field correction using the IRAF software package. Extraction (task extractor), including background sky subtraction, of data allowed the creation of one-dimensional spectra, wavelength calibrated using standard lines from a $\mathrm{Cu}-\mathrm{Ar}$ arc. Observing conditions were not photometric, seeing was limited to $2^{\prime \prime}$ at best but varied throughout the evening. We show in Fig. 4 the resulting spectrum: its moderate signal-to-noise ratio does not allow us to make a spectral classification, but a significant $\mathrm{H}_{\alpha}$ emission line, with equivalent width $E W=-37.44 \AA$, is clearly visible.

Despite no periodicities being found in the OGLE light curve of this star, these findings indicate that source \#13 is a Be HMXRB. Since sources of this class typically have a transient nature, we searched for hard X-ray outbursts in all the public data obtained with the INTEGRAL satellite (Winkler et al. 2003), that is all pointings obtained with the IBIS instrument (Ubertini et al. 2003) in which the source position was within 


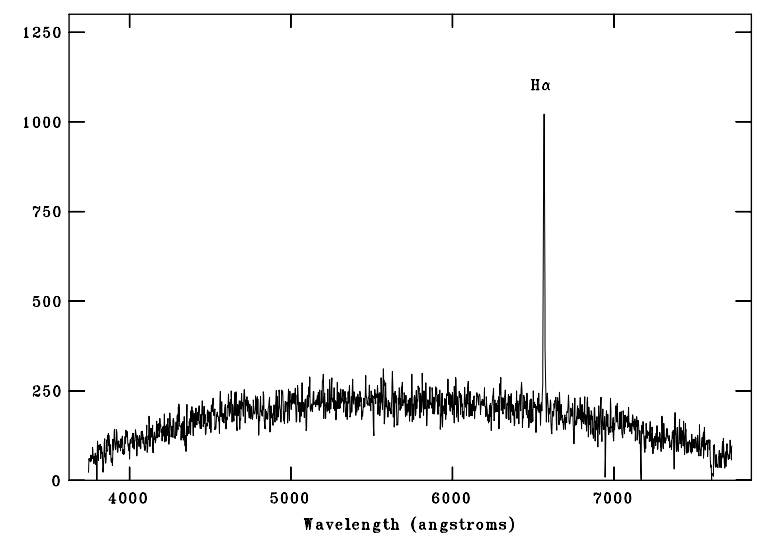

Fig. 4. SAAO optical spectrum of source \#13; note the presence of a strong $\mathrm{H}_{\alpha}$ emission line.

12 degrees from the center of the field of view. This led to a total of 619 pointings, with different exposure times from about $1800 \mathrm{~s}$ to about $3600 \mathrm{~s}$, spanning from July 2003 to June 2009. We used version 9.0 of the Off-line Scientific Analysis software to analyse the data. For each pointing we extracted an image in the 17-40 keV energy band, but in none of them was the source significantly detected. The typical flux upper limit of the individual images is on the order of $\sim 20 \mathrm{mCrab}$. We also produced a deep image by summing all the individal pointings, reaching a total exposure of about $2 \mathrm{Ms}$. The source was not detected, with a $5 \sigma$ upper limit of about $1 \mathrm{mCrab}$ in the $17-40 \mathrm{keV}$ range, which is well above the extrapolation of the flux seen by EPIC.

\section{Discussion}

We have detected 30 sources with hardness ratios indicating a column density $N_{\mathrm{H}}>3 \times 10^{23} \mathrm{~cm}^{-2}$. Five of these sources are HMXRBs, of which one was discovered in our search and spectroscopically identified as a new Be binary system. Most of the remaining 25 sources have very faint $(V>18)$ or no optical counterparts, with colors not compatible with reddened early type-stars (unless a significant IR excess is present). Therefore, they can hardly be considered as candidate absorbed HMXRBs.

Spectral information for a few of the brightest sources of our sample was previously reported in other works. The XMM-Newton spectrum of the $25 \mathrm{~s}$ pulsar XMMU J004814.1-731003 was closely fit with a power law of photon index $1.33 \pm 0.27$ and $N_{\mathrm{H}}=5 \times 10^{22} \mathrm{~cm}^{-2}$, confirming our conclusion that it is a highly absorbed source. Our brightest source (\#18) is the 293 s pulsar RX J0058.2-7231, for which Haberl et al. (2008a) reported an absorption $N_{\mathrm{H}}=(1.35 \pm 0.32) \times 10^{22} \mathrm{~cm}^{-2}$, considerably smaller than the value we inferred from the hardness ratio. This might be due to the results of Haberl et al. (2008a) being obtained from the June 2007 XMM-Newton observation, while our results for this source are based on the SMC Survey pointing carried out in October 2009, when the source was about a factor of ten brighter. HMXRBs can display large variations in their intrinsic absorption, as shown for example in the case of the long period (1323 s) SMC pulsar RXJ0103.6-7201 (Eger \& Haberl 2008).

We do not expect of course all the sources in our sample to be HMXRBs in the SMC. A large fraction of them will turn out to be background-absorbed AGNs. On the other, hand our sample should not contain foreground stars belonging to our Galaxy, which would show softer and less absorbed X-ray spectra.
A rough estimate of the number of absorbed AGNs in our sample can be derived using published number flux relations for extragalactic sources. For example, based on the $\log N-$ $\log S$ relations for AGNs with $N_{\mathrm{H}}=3 \times 10^{23} \mathrm{~cm}^{-2}$ by Gilli et al. (2007), we obtain a surface density of $\sim 4$ AGNs per square degree at a flux limit of $4 \times 10^{-14} \mathrm{erg} \mathrm{cm}^{-2} \mathrm{~s}^{-1}$. Our survey covered a sky area of $\sim 5$ square degrees, although with a non-uniform sensitivity, because of telescope vignetting and different exposure times. Owing to the uncertainties in the $\log N-\log S$ relations and the small number of objects, this prediction is consistent with the number of unidentified sources in our sample.

\section{Conclusions}

Motivated by the presence of several highly absorbed HMXRBs in our Galaxy, we have carried out a search for similar systems in the SMC exploiting the nearly complete coverage of this galaxy obtained with XMM-Newton. Our selection criteria, corresponding to an absorption threshold of $\sim 3 \times 10^{23} \mathrm{~cm}^{-2}$, were met by four known HMXRBs and led to the discovery of a new highly absorbed Be binary in the SMC. We also selected other 25 additional sources, among which other highly absorbed HMXRBs might be present. Since we also expect a good fraction of these sources to be AGNs, we can say that the SMC does not contain much more than $\sim 10$ persistent HMXRBs with intrinsic $N_{\mathrm{H}}>3 \times 10^{23} \mathrm{~cm}^{-2}$. We cannot exclude the presence of a much larger population of intrinsically absorbed binaries of transient nature, which could not be detected in our survey if their quiescent luminosity is below a few $10^{34} \mathrm{erg} \mathrm{s}^{-1}$.

Acknowledgements. We wish to thank Lara Sidoli for the useful discussion. This work is based on observations obtained with XMM-Newton, an ESA science mission with instruments and contributions directly funded by ESA Member States and NASA. The XMM-Newton data analysis is supported by the Italian Space Agency (ASI) (ASI/INAF contract I/032/10/0). We were granted observation time at the South African Astronomical Observatory (SAAO) and wish to thank them for their kind help and accommodations. Travel to the SAAO was funded by Australian Government AINSTO AMNRF grant number 10/11-O-06. Partly based on observations with INTEGRAL, an ESA project with instruments and science data centre funded by ESA member states (especially the PI countries: Denmark, France, Germany, Italy, Spain, and Switzerland), Czech Republic and Poland, and with the participation of Russia and the USA. IRAF is the Image Reduction and Analysis Facility, a general purpose software system for the reduction and analysis of astronomical data. IRAF is written and supported by the IRAF programming group at the National Optical Astronomy Observatories (NOAO) in Tucson, Arizona. NOAO is operated by the Association of Universities for Research in Astronomy (AURA), Inc. under cooperative agreement with the National Science Foundation. Extractor is part of an IRAF package (PNDR) developed to assist with the efficient reduction of long-slit spectra of emission line objects. It was developed as part of the Macquarie/AAO/Strasbourg Halpha (MASH) Planetary Nebulae Catalogue.

\section{References}

Antoniou, V., Zezas, A., Hatzidimitriou, D., \& McDowell, J. C. 2009, ApJ, 697, 1695

Antoniou, V., Zezas, A., Hatzidimitriou, D., \& Kalogera, V. 2010, ApJ, 716, L140

Baldi, A., Molendi, S., Comastri, A., et al. 2002, ApJ, 564, 190

Bird, A. J., Coe, M. J., \& McBride, V. 2011, MNRAS, submitted

Coe, M. J., \& Edge, W. R. T. 2004, MNRAS, 350, 756

Eger, P., \& Haberl, F. 2008, A\&A, 491, 841

Galache, J. L., Corbet, R. H. D., Coe, M. J., et al. 2008, ApJS, 177, 189

Gilli, R., Comastri, A., \& Hasinger, G. 2007, A\&A, 463, 79

Haberl, F., \& Pietsch, W. 2008, in proc. ESAC faculty workshop on X-rays From Nearby Galaxies, 32

Haberl, F., Eger, P., \& Pietsch, W. 2008a, A\&A, 489, 327

Haberl, F., Eger, P., Pietsch, W., Corbet, R. H. D., \& Sasaki, M. 2008b, A\&A, 485,177

Hatziminaoglou, E., Groenewegen, M. A. T., da Costa, L., et al. 2002, A\&A, 384,81 
G. Novara et al.: Highly-absorbed X-ray binaries in the SMC

Hilditch, R. W., Harries, T. J., \& Howarth, I. D. 2005, Highlights of Astronomy, 13,455

Johnson, H. L. 1966, ARA\&A, 4, 193

Kato, D., Nagashima, C., Nagayama, T., et al. 2007, PASJ, 59, 615

Kouzuma, S., \& Yamaoka, H. 2010, A\&A, 509, A64

Laycock, S., Zezas, A., Hong, J., Drake, J. J., \& Antoniou, V. 2010, ApJ, 716, 1217

Liu, Q. Z., van Paradijs, J., \& van den Heuvel, E. P. J. 2005, A\&A, 442, 1135

Liu, Q. Z., van Paradijs, J., \& van den Heuvel, E. P. J. 2006, VizieR Online Data Catalog, 345, 51165

Macomb, D. J., Fox, D. W., Lamb, R. C., \& Prince, T. A. 2003, ApJ, 584, L79

Maeder, A., Grebel, E. K., \& Mermilliod, J.-C. 1999, A\&A, 346, 459

Majid, W. A., Lamb, R. C., \& Macomb, D. J. 2004, ApJ, 609, 133

McBride, V. A., Coe, M. J., Negueruela, I., Schurch, M. P. E., \& McGowan, K. E. 2008, MNRAS, 388, 1198

McGowan, K. E., Coe, M. J., Schurch, M. P. E., et al. 2008, MNRAS, 383, 330

Novara, G., La Palombara, N., Carangelo, N., et al. 2006, A\&A, 448, 93
Rajoelimanana, A. F., Charles, P. A., \& Udalski, A. 2011, MNRAS, 413, 1600

Russell, S. C., \& Dopita, M. A. 1992, ApJ, 384, 508

Sasaki, M., Pietsch, W., \& Haberl, F. 2003, A\&A, 403, 901

Schmidtke, P. C., Cowley, A. P., Levenson, L., \& Sweet, K. 2004, AJ, 127, 3388

Shtykovskiy, P., \& Gilfanov, M. 2005, MNRAS, 362, 879

Shtykovskiy, P. E., \& Gilfanov, M. R. 2007, Astron. Lett., 33, 437

Sidoli, L. 2010, AIP Conf. Ser., 1314, 271

Stanimirovic, S., Staveley-Smith, L., Dickey, J. M., Sault, R. J., \& Snowden, S. L. 1999, MNRAS, 302, 417

Strüder, L., Briel, U., Dennerl, K., et al. 2001, A\&A, 365, L18

Turner, M. J. L., Abbey, A., Arnaud, M., et al. 2001, A\&A, 365, L27

Ubertini, P., Lebrun, F., Di Cocco, G., et al. 2003, A\&A, 411, L131

Wilms, J., Allen, A., \& McCray, R. 2000, ApJ, 542, 914

Winkler, C., Courvoisier, T. J.-L., Di Cocco, G., et al. 2003, A\&A, 411, L1

Zaritsky, D., Harris, J., Thompson, I. B., Grebel, E. K., \& Massey, P. 2002, AJ, 123,855 
Table 1. Log of the XMM-Newton observations of 62 SMC fields with the corresponding net good time interval (GTI) for the two MOS and the $p n$ cameras.

\begin{tabular}{|c|c|c|c|c|c|}
\hline \multirow[t]{2}{*}{ Observation ID } & \multirow{2}{*}{$\begin{array}{l}\text { XMM-Newton } \\
\text { revolution }\end{array}$} & \multirow{2}{*}{$\begin{array}{l}\text { Date } \\
\text { (UT) }\end{array}$} & \multicolumn{3}{|c|}{ GTI (ks) } \\
\hline & & & MOS1 & MOS2 & $p n$ \\
\hline 0011450101 & 0270 & 2001-05-31T02:20:10 & 42.8 & 43.0 & 40.8 \\
\hline 0011450201 & 0355 & 2001-11-16Т03:23:32 & 37.2 & 38.5 & 40.1 \\
\hline 0018540101 & 0357 & 2001-11-20T23:42:37 & 26.7 & 27.0 & 23.4 \\
\hline 0084200101 & 0422 & 2002-03-30T13:48:28 & 8.8 & 8.2 & 7.1 \\
\hline 0084200801 & 0340 & $2001-10-17 \mathrm{~T} 10: 07: 40$ & 20.7 & 20.7 & 17.1 \\
\hline 0110000101 & 0156 & $2000-10-15 T 15: 18: 28$ & 25.2 & 25.7 & 14.7 \\
\hline 0110000301 & 0157 & $2000-10-17 \mathrm{~T} 21: 45: 54$ & 12.6 & 12.4 & 7.4 \\
\hline 0112780201 & 0143 & 2000-09-19Т02:05:37 & 3.6 & 2.3 & 0.0 \\
\hline 0112780601 & 0254 & 2001-04-29T21:07:59 & 0.9 & 1.5 & 2.9 \\
\hline 0135721701 & 0721 & 2003-11-16T06:12:02 & 24.4 & 24.8 & 29.8 \\
\hline 0142660801 & 0721 & 2003-11-17T03:55:54 & 7.2 & 7.5 & 6.8 \\
\hline 0157960201 & 0737 & $2003-12-18 \mathrm{~T} 14: 32: 45$ & 17.9 & 18.7 & 17.2 \\
\hline 0164560401 & 0803 & 2004-04-28T22:08:04 & 0.2 & 0.1 & 0.1 \\
\hline 0301170101 & 1151 & 2006-03-22T21:39:54 & 18.8 & 19.7 & 16.4 \\
\hline 0301170201 & 1151 & 2006-03-23Т04:48:17 & 22.5 & 22.7 & 14.4 \\
\hline 0301170301 & 1158 & 2006-04-06Т04:32:35 & 17.9 & 18.0 & 14.4 \\
\hline 0301170601 & 1153 & 2006-03-27T12:21:01 & 14.4 & 14.5 & 11.7 \\
\hline 0311590601 & 1146 & 2006-03-13T15:17:13 & 11.2 & 11.0 & 9.6 \\
\hline 0402000101 & 1248 & 2006-10-03Т00:09:09 & 21.7 & 21.7 & 19.0 \\
\hline 0403970301 & 1329 & 2007-03-12Т20:02:20 & 28.0 & 28.7 & 19.2 \\
\hline 0404680201 & 1263 & 2006-11-01T00:56:29 & 32.4 & 32.4 & 30.8 \\
\hline 0404680301 & 1344 & 2007-04-11T19:38:25 & 20.1 & 19.9 & 15.4 \\
\hline 0404680501 & 1344 & 2007-04-12T03:07:23 & 23.3 & 23.7 & 21.6 \\
\hline 0500980101 & 1380 & 2007-06-23Т05:51:39 & 25.2 & 25.0 & 22.3 \\
\hline 0500980201 & 1372 & 2007-06-06T08:52:16 & 27.6 & 28.3 & 14.1 \\
\hline 0501470101 & 1371 & 2007-06-04T08:59:50 & 13.4 & 16.5 & 9.6 \\
\hline 0503000201 & 1444 & 2007-10-28T05:49:58 & 21.5 & 21.5 & 20.0 \\
\hline 0503000301 & 1514 & 2008-03-16T15:25:16 & 17.9 & 19.3 & 2.0 \\
\hline 0601210101 & 1727 & 2009-05-14T09:14:24 & 20.7 & 21.2 & 19.8 \\
\hline 0601210201 & 1794 & 2009-09-25Т00:15:42 & 37.5 & 37.5 & 35.7 \\
\hline 0601210301 & 1729 & 2009-05-18T10:29:15 & 30.9 & 31.4 & 27.5 \\
\hline 0601210401 & 1794 & 2009-09-25Т11:22:24 & 37.6 & 37.6 & 36.0 \\
\hline 0601210501 & 1794 & 2009-09-25T22:30:43 & 49.8 & 50.4 & 39.9 \\
\hline 0601210601 & 1795 & 2009-09-27Т00:10:53 & 36.7 & 37.1 & 32.7 \\
\hline 0601210701 & 1795 & 2009-09-27T11:35:54 & 38.7 & 38.6 & 37.1 \\
\hline 0601210801 & 1801 & 2009-10-09T18:33:30 & 24.7 & 24.7 & 23.1 \\
\hline 0601210901 & 1795 & 2009-09-27T23:02:34 & 34.9 & 35.3 & 33.9 \\
\hline 0601211001 & 1817 & 2009-11-09T21:16:08 & 34.0 & 35.0 & 23.7 \\
\hline 0601211101 & 1806 & 2009-10-18T22:47:09 & 31.6 & 31.6 & 30.1 \\
\hline 0601211201 & 1807 & 2009-10-20T22:51:09 & 33.1 & 33.2 & 29.0 \\
\hline 0601211301 & 1798 & 2009-10-03Т05:08:47 & 32.4 & 32.5 & 30.9 \\
\hline 0601211401 & 1814 & 2009-11-04T21:38:31 & 35.3 & 35.6 & 31.1 \\
\hline 0601211501 & 1803 & 2009-10-13Т00:02:01 & 37.6 & 37.4 & 34.0 \\
\hline 0601211601 & 1802 & 2009-10-11T22:43:47 & 31.6 & 32.3 & 28.8 \\
\hline 0601211701 & 1804 & 2009-10-16Т01:05:21 & 25.1 & 26.6 & 18.4 \\
\hline 0601211801 & 1819 & 2009-11-13Т20:59:56 & 26.2 & 30.2 & 22.3 \\
\hline 0601211901 & 1827 & 2009-11-30T14:46:19 & 31.7 & 31.7 & 30.1 \\
\hline 0601212001 & 1826 & 2009-11-27T22:39:06 & 28.3 & 28.3 & 26.3 \\
\hline 0601212101 & 1820 & 2009-11-16T05:48:08 & 34.1 & 34.1 & 32.5 \\
\hline 0601212201 & 1822 & 2009-11-16T06:10:27 & 27.1 & 29.4 & 24.3 \\
\hline 0601212301 & 1786 & 2009-09-09Т09:13:53 & 33.5 & 33.5 & 31.9 \\
\hline 0601212401 & 1750 & 2009-06-29T14:46:19 & 29.5 & 30.9 & 23.9 \\
\hline 0601212501 & 1786 & 2009-09-09T19:13:54 & 33.5 & 33.5 & 31.9 \\
\hline 0601212601 & 1750 & 2009-06-29T06:04:39 & 26.0 & 27.1 & 17.5 \\
\hline 0601212701 & 1840 & 2009-12-26Т07:25:22 & 36.7 & 36.7 & 34.5 \\
\hline 0601212801 & 1831 & 2009-12-07T23:35:54 & 21.3 & 21.3 & 16.7 \\
\hline 0601212901 & 1788 & 2009-09-13Т13:29:26 & 36.1 & 36.1 & 34.5 \\
\hline 0601213001 & 1788 & 2009-09-13Т01:11:03 & 41.6 & 41.7 & 38.9 \\
\hline 0601213201 & 1878 & 2010-03-12Т00:56:15 & 12.5 & 13.2 & 9.4 \\
\hline 0601213301 & 1878 & 2010-03-12Т05:26:15 & 9.8 & 9.9 & 8.1 \\
\hline 0601213401 & 1880 & 2010-03-16T10:05:12 & 17.6 & 17.5 & 11.9 \\
\hline 0656780201 & 1886 & 2010-03-27T12:20:41 & 11.0 & 12.3 & 7.0 \\
\hline
\end{tabular}

